\title{
Global validation of the WSES Sepsis Severity Score for patients with complicated intra-abdominal infections: a prospective multicentre study (WISS Study)
}

Massimo Sartelli ${ }^{1 *}$, Fikri M. Abu-Zidan², Fausto Catena ${ }^{3}$, Ewen A. Griffiths ${ }^{4}$, Salomone Di Saverio ${ }^{5}$, Raul Coimbra ${ }^{6}$, Carlos A. Ordoñez ${ }^{7}$, Ari Leppaniemi ${ }^{8}$, Gustavo P. Fraga ${ }^{9}$, Federico Coccolini ${ }^{10}$, Ferdinando Agresta ${ }^{11}$, Asrhaf Abbas ${ }^{12}$, Saleh Abdel Kader ${ }^{13}$, John Agboola ${ }^{14}$, Adamu Amhed ${ }^{15}$, Adesina Ajibade ${ }^{16}$, Seckin Akkucuk ${ }^{17}$, Bandar Alharthi ${ }^{18}$, Dimitrios Anyfantakis ${ }^{19}$, Goran Augustin ${ }^{20}$, Gianluca Baiocchi ${ }^{21}$, Miklosh Bala ${ }^{22}$, Oussama Baraket ${ }^{23}$, Savas Bayrak ${ }^{24}$, Giovanni Bellanova ${ }^{25}$, Marcelo A. Beltràn ${ }^{26}$, Roberto Bini ${ }^{27}$, Matthew Boal ${ }^{4}$, Andrey V. Borodach ${ }^{28}$, Konstantinos Bouliaris ${ }^{29}$, Frederic Branger ${ }^{30}$, Daniele Brunelli ${ }^{31}$, Marco Catani ${ }^{32}$, Asri Che Jusoh ${ }^{33}$, Alain Chichom-Mefire ${ }^{34}$, Gianfranco Cocorullo ${ }^{35}$, Elif Colak ${ }^{36}$, David Costa ${ }^{37}$, Silvia Costa ${ }^{38}$, Yunfeng Cuii ${ }^{39}$, Geanina Loredana Curca ${ }^{40}$, Terry Curry ${ }^{6}$, Koray Das ${ }^{41}$, Samir Delibegovic ${ }^{42}$, Zaza Demetrashvili ${ }^{43}$, Isidoro Di Carlo ${ }^{44}$, Nadezda Drozdova ${ }^{45}$, Tamer El Zalabany ${ }^{46}$, Mushira Abdulaziz Enani ${ }^{47}$, Mario Faro ${ }^{48}$, Mahir Gachabayov ${ }^{49}$, Teresa Giménez Maurel ${ }^{50}$, Georgios Gkiokas ${ }^{51}$, Carlos Augusto Gomes ${ }^{52}$, Ricardo Alessandro Teixeira Gonsaga ${ }^{53}$, Gianluca Guercioni ${ }^{54}$, Ali Guner ${ }^{55}$, Sanjay Gupta ${ }^{56}$, Sandra Gutierrez ${ }^{57}$, Martin Hutan ${ }^{58}$, Orestis loannidis ${ }^{59}$, Arda Isik ${ }^{60}$, Yoshimitsu Izawa ${ }^{61}$, Sumita A. Jain ${ }^{62}$, Mantas Jokubauskas ${ }^{63}$, Aleksandar Karamarkovic ${ }^{64}$, Saila Kauhanen ${ }^{65}$, Robin Kaushik ${ }^{56}$, Jakub Kenig ${ }^{66}$, Vladimir Khokha ${ }^{67}$, Jae II Kim ${ }^{68}$, Victor Kong ${ }^{69}$, Renol Koshy ${ }^{44}$, Avidyl Krasniqii ${ }^{70}$, Ashok Kshirsagar ${ }^{71}$, Zygimantas Kuliesius ${ }^{72}$, Konstantinos Lasithiotakis ${ }^{73}$, Pedro Leão ${ }^{74}$, Jae Gil Lee ${ }^{75}$, Miguel Leon ${ }^{76}$, Aintzane Lizarazu Pérez ${ }^{77}$, Varut Lohsiriwat ${ }^{78}$, Eudaldo López-Tomassetti Fernandez ${ }^{79}$, Eftychios Lostoridis ${ }^{80}$, Raghuveer Mn ${ }^{81}$, Piotr Major ${ }^{82}$, Athanasios Marinis ${ }^{83}$, Daniele Marrelli ${ }^{84}$, Aleix Martinez-Perez ${ }^{85}$, Sanjay Marwah ${ }^{86}$, Michael McFarlane ${ }^{87}$, Renato Bessa Melo ${ }^{88}$, Cristian Mesina ${ }^{89}$, Nick Michalopoulos ${ }^{90}$, Radu Moldovanu ${ }^{91}$, Ouadii Mouagit ${ }^{92}$, Akutu Munyika ${ }^{93}$, Ionut Negoi ${ }^{94}$, loannis Nikolopoulos ${ }^{95}$, Gabriela Elisa Nita ${ }^{10}$, Iyiade Olaoye ${ }^{96}$, Abdelkarim Omari ${ }^{97}$, Paola Rodríguez Ossa ${ }^{7}$, Zeynep Ozkan ${ }^{98}$, Ramakrishnapillai Padmakumar ${ }^{99}$, Francesco Pata ${ }^{100}$, Gerson Alves Pereira Junior ${ }^{101}$, Jorge Pereira ${ }^{102}$, Tadeja Pintar ${ }^{103}$, Konstantinos Pouggouras ${ }^{80}$, Vinod Prabhu ${ }^{104}$, Stefano Rausei ${ }^{105}$, Miran Rems ${ }^{106}$, Daniel Rios-Cruz ${ }^{107}$, Boris Sakakushev ${ }^{108}$, Maria Luisa Sánchez de Molina ${ }^{109}$, Charampolos Seretis ${ }^{110}$, Vishal Shelat ${ }^{111}$, Romeo Lages Simões ${ }^{9}$, Giovanni Sinibaldi ${ }^{112}$, Matej Skrovina ${ }^{113}$, Dmitry Smirnov ${ }^{114}$, Charalampos Spyropoulos ${ }^{115}$, Jaan Tepp ${ }^{116}$, Tugan Tezcaner ${ }^{117}$, Matti Tolonen ${ }^{8}$, Myftar Torba $^{118}$, Jan Ulrych ${ }^{119}$, Mustafa Yener Uzunoglu ${ }^{120}$, David van Dellen ${ }^{121}$, Gabrielle H. van Ramshorst ${ }^{122}$, Giorgio Vasquez ${ }^{123}$, Aurélien Venara ${ }^{30}$, Andras Vereczkei ${ }^{124}$, Nereo Vettoretto ${ }^{125}$, Nutu Vlad ${ }^{126}$, Sanjay Kumar Yadav ${ }^{127}$, Tonguç Utku Yilmaz $^{128}$, Kuo-Ching Yuan ${ }^{129}$, Sanoop Koshy Zachariah ${ }^{130}$, Maurice Zida ${ }^{131}$, Justas Zilinskas ${ }^{63}$ and Luca Ansaloni ${ }^{10}$

\footnotetext{
* Correspondence: massimosartelli@gmail.com

${ }^{1}$ Department of Surgery, Macerata Hospital, Macerata, Italy

Full list of author information is available at the end of the article
} 


\begin{abstract}
Background: To validate a new practical Sepsis Severity Score for patients with complicated intra-abdominal infections (CIAls) including the clinical conditions at the admission (severe sepsis/septic shock), the origin of the CIAls, the delay in source control, the setting of acquisition and any risk factors such as age and immunosuppression.

Methods: The WISS study (WSES cIAls Score Study) is a multicenter observational study underwent in 132 medical institutions worldwide during a four-month study period (October 2014-February 2015). Four thousand five hundred thirty-three patients with a mean age of 51.2 years (range 18-99) were enrolled in the WISS study.

Results: Univariate analysis has shown that all factors that were previously included in the WSES Sepsis Severity Score were highly statistically significant between those who died and those who survived $(p<0.0001)$. The multivariate logistic regression model was highly significant $(p<0.0001, \mathrm{R} 2=0.54)$ and showed that all these factors were independent in predicting mortality of sepsis. Receiver Operator Curve has shown that the WSES Severity Sepsis Score had an excellent prediction for mortality. A score above 5.5 was the best predictor of mortality having a sensitivity of $89.2 \%$, a specificity of $83.5 \%$ and a positive likelihood ratio of 5.4 .
\end{abstract}

Conclusions: WSES Sepsis Severity Score for patients with complicated Intra-abdominal infections can be used on global level. It has shown high sensitivity, specificity, and likelihood ratio that may help us in making clinical decisions.

Keywords: Intra-abdominal, Infections, Sepsis, Septic shock

\section{Background}

Intra-abdominal infections (IAIs) include several different pathological conditions [1] and are usually classified into uncomplicated and complicated. In complicated IAIs (cIAIs), the infectious process extends beyond the organ, and causes either localized peritonitis or diffuse peritonitis. The treatment of patients with complicated intra-abdominal infections involves both source control and antibiotic therapy. Complicated IAIs are an important cause of morbidity and may be associated with poor prognosis. However the term "complicated intra-abdominal infections" describes a wide heterogeneity of patient populations, making it difficult to suggest a general treatment regimen and stressing the need of an individualized approach to decision making.

Early prognostic evaluation of complicated intraabdominal infections is crucial to assess the severity and decide the aggressiveness of treatment. Many factors influencing the prognosis of patients with cIAIs have been described, including advanced age, poor nutrition, pre-existing diseases, immunosuppression, extended peritonitis, occurrence of septic shock, poor source control, organ failures, prolonged hospitalization before therapy, and infection with nosocomial pathogens [2-10].

Recently the World Society of Emergency Surgery (WSES) designed a global prospective observational study (CIAOW Study) [11, 12]. All the risk factors for occurrence of death during hospitalization were evaluated and then discussed with an international panel of experts. The most significant variables, adjusted to clinical criteria, were used to create a severity score for patients with cIAIs including the clinical conditions at admission (severe sepsis/septic shock), the origin of the cIAIs, the delay in source control, the setting of acquisition and any risk factors such as age and immunosuppression (Appendix).

There may be different causes of sepsis, health care standards, and differences in underlying health status, economical differences that make prediction of sepsis on global level difficult. The WSES addressed this issue in the present study which aims to validate a previous score on a global level.

\section{Methods}

Ethical statement

The study met the standards outlined in the Declaration of Helsinki and Good Epidemiological Practices. This study did not change or modify the laboratory or clinical practices of each centre and differences of practices were kept as they are. The data collection was anonymous and identifiable patient information was not submitted.

Individual researchers were responsible for complying with local ethical standards and hospital registration of the study.

\section{Study population}

This multicenter observational study was run in 132 medical institutions from 54 countries worldwide during a four-month period (October 2014-February 2015). Inclusion criteria were patients older than 18 years with complicated intra-abdominal sepsis (cIAIs) who had surgical management or interventional radiological drainage. cIAIs was defined as an infectious process that proceeded beyond the organ, and caused either localized peritonitis/abscess or diffuse peritonitis [13]. Patients who were younger than 18 years, or those 
who had pancreatitis, or primary peritonitis were excluded from the study. Severe sepsis was defined as sepsis-induced tissue hypoperfusion or organ dysfunction (any of the following thought to be due to the infection): hypotension $(<90 / 60$ or MAP $<65)$, lactate above upper limits laboratory normal, Urine output $<0.5 \mathrm{~mL} / \mathrm{kg} /$ $\mathrm{h}$ for more than $2 \mathrm{~h}$ despite adequate fluid resuscitation, Creatinine $>2.0 \mathrm{mg} / \mathrm{dL}(176.8 \mu \mathrm{mol} / \mathrm{L})$, Bilirubin $>2 \mathrm{mg} / \mathrm{dL}$ $(34.2 \mu \mathrm{mol} / \mathrm{L})$, Platelet count $<100,000 \mu \mathrm{L}$, Coagulopathy (international normalized ratio $>1.5$ ), Acute lung injury with Pao2/Fio2 $<250$ in the absence of pneumonia as infection source. Septic shock was defined as severe sepsis associated with refractory hypotension $(\mathrm{BP}<90 / 60)$ despite adequate fluid resuscitation [14].

WSES Sepsis Severity Score for patients with complicated Intra-abdominal infections is shown in Appendix.

\section{Data monitoring and collection}

The study was monitored by the coordination center, which investigated and verified missing or unclear data submitted to the central database. This study was performed under the direct supervision of the Board of Directors of WSES. In each centre, the coordinator collected and compiled data in an online case report system. Data were entered directly through a web-based computerized database. Data were entered either by a drop menu for categorical data like the source of infection or numbers for continuous variables such as age. Data collected included demographic data of the patient and disease characteristics, demographical data, type of infection (community- or healthcare-acquired), severity criteria and origin of infection and surgical procedures performed.

\section{Statistical analysis}

Sepsis status was coded as ordinal data for testing the logistic regression (not for scoring) as follows: no sepsis $=0$, sepsis $=2$, severe sepsis $=3$, septic shock $=4$ ). The source of sepsis was analysed as categorical data in the logistic regression, and the age as continuous data, while healthcare associated infection, delay in management, and immunosuppression as binomial data. The variables used in this scoring system in the patients who survived and those who died were compared using univariate analysis. This included Fisher's exact test or Pearson Chi-Square as appropriate for categorical data and Mann-Whitney U-test for continuous or ordinal data. Significant factors were then entered into a direct logistic regression model. A p value of $\leq 0.05$ was considered significant. Data were analyzed with PASW Statistics 21, SPSS Inc, USA.

\section{Results}

Four thousand six hundred fifty-two cases were collected in the online case report system. One hundred twentynine cases did not meet the inclusion criteria. Four thousand five hundred thirty-three patients with a mean age of 51.2 years (range 18-99) were enrolled in the WISS study. One thousand nine hundred thirty-five patients $(42.7 \%)$ were women and 2598 (57.3\%) were men.

Among these patients, 3966 (87.5 \%) were affected by community-acquired IAIs while the remaining 567 $(12.5 \%)$ suffered from healthcare-associated infections. One thousand six hundred twenty-seven patients (35.9\%) were affected by generalized peritonitis while 2906 (64.1 \%) suffered from localized peritonitis or abscesses. Seven hundred ninety-one patients (17.4 \%) were admitted in critical condition (severe sepsis/septic shock). The various sources of infection are outlined in Table 1 . The most frequent source of infection was acute appendicitis; 1553 cases (34.2\%) involved complicated appendicitis.

The overall mortality rate was $9.2 \%$ (416/4533).

Table 2 shows the univariate analysis comparing patients with complicated intra-abdominal infection who survived and those who died. The analysis shows that all factors included in the Sepsis Severity Score were highly significantly different between those who died and those who survived ( $p<0.0001$ in all variables). Accordingly all factors were entered into a direct logistic regression model (Table 3 ). The direct logistic regression model was highly significant $(p<0.0001$, $\mathrm{R} 2=0.54$ ) and showed that all factors included in the Sepsis Severity Score were significant independent

Table 1 Source of infection in 4553 patients from 132 hospitals worldwide (15 October 2014-15 February 2015)

\begin{tabular}{ll}
\hline Source of infection & Number (\%) \\
\hline Appendicitis & $1553(34.2 \%)$ \\
Cholecystitis & $837(18.5 \%)$ \\
Post-operative & $387(8.5 \%)$ \\
Colonic non diverticular perforation & $269(5.9 \%)$ \\
Gastro-duodenal perforations & $498(11 \%)$ \\
Diverticulitis & $234(5.2 \%)$ \\
Small bowel perforation & $243(5.4 \%)$ \\
Others & $348(7.7 \%)$ \\
PID & $50(1.1 \%)$ \\
Post traumatic perforation & $114(2.5 \%)$ \\
Missing & \\
Total & $4553(100 \%)$ \\
\hline
\end{tabular}

PID pelvic inflammatory disease 
Table 2 Univariate analysis of patients with complicated intra-abdominal infection comparing patients who survived ( $n=4117)$ and patient who died $(n=416)$

\begin{tabular}{|c|c|c|c|}
\hline Variable & Survided (\%) $n=4117$ & Died (\%) $n=416$ & $p$ value \\
\hline Sepsis status & & & $<0.0001$ \\
\hline No sepsis & 1914 (46.5 \%) & $23(5.5 \%)$ & \\
\hline Sepsis & 1725 (41.9 \%) & $80(19.2 \%)$ & \\
\hline Severe sepsis & $404(9.8 \%)$ & $157(37.7 \%)$ & \\
\hline Septic shock & $74(1.8 \%)$ & $156(37.5 \%)$ & \\
\hline Healthcare associated infection & $433(10.5 \%)$ & $134(32.2 \%)$ & $<0.0001$ \\
\hline Source of infection & & & $<0.0001$ \\
\hline Appendicitis & $1536(37.3 \%)$ & $17(4.1 \%)$ & \\
\hline Cholecystitis & $809(19.7 \%)$ & 28 (6.7 \%) & \\
\hline Colonic non diverticular perforation & $204(5 \%)$ & $65(15.6 \%)$ & \\
\hline Diverticulitis & $203(4.9 \%)$ & $31(7.5 \%)$ & \\
\hline Gastro-duodenal perforation & $431(10.5 \%)$ & $67(16.2 \%)$ & \\
\hline PID & $50(1.2 \%)$ & $0(0)$ & \\
\hline Postoperative & $415(10.1 \%)$ & $86(20.7 \%)$ & \\
\hline Small bowel perforation & $174(4.2 \%)$ & $69(16.6 \%)$ & \\
\hline Post-traumatic & $104(2.5 \%)$ & $10(2.4 \%)$ & \\
\hline Others & $259(6.3 \%)$ & $53(12.7 \%)$ & \\
\hline Delay in source control & 2015 (48.9 \%) & 341 (82 \%) & $<0.0001$ \\
\hline Median age years (range) & $48(18-97)$ & 79 (18-99) & $<0.0001$ \\
\hline Immunosuppresion & $292(7.1)$ & $120(28.8 \%)$ & $<0.0001$ \\
\hline Sepsis severity score & $3(0-17)$ & $10(0-17)$ & $<0.0001$ \\
\hline
\end{tabular}

Data presented as median range or number percentage as appropriate

$P I D$ pelvic inflammatory disease

$p$ value $=$ Fisher's exact test, Pearson Chi-Square, or Mann Whitney $U$ test as appropriate

Table 3 Direct logistic regression model with factors affecting mortality of patients complicated intra-abdominal infection, global study of 132 centres, $(n=4553)$

\begin{tabular}{|c|c|c|c|c|c|c|c|}
\hline \multirow[t]{2}{*}{ Score variable } & \multirow[t]{2}{*}{ B } & \multirow[t]{2}{*}{ S.E. } & \multirow[t]{2}{*}{ Wald test } & \multirow[t]{2}{*}{$P$ value } & \multirow[t]{2}{*}{ OR } & \multicolumn{2}{|c|}{ OR 95 \% C.I. } \\
\hline & & & & & & Lower & Upper \\
\hline Sepsis status & 1.57 & 0.08 & 365.59 & $<0.0001$ & 4.81 & 4.09 & 5.65 \\
\hline Setting of infection acquisition & 0.6 & 0.18 & 10.49 & 0.001 & 1.81 & 1.27 & 2.6 \\
\hline Source of infection ${ }^{a}$ & & & 59.38 & $<0.0001$ & & & \\
\hline Colonic non-diverticulical perforation & -0.26 & 0.27 & 0.97 & 0.33 & 0.77 & 0.46 & 1.3 \\
\hline Diverticulitis diffuse peritonitis & -0.26 & 0.34 & 0.51 & 0.48 & 0.78 & 0.40 & 1.54 \\
\hline Postoperative diffuse peritonitis & -0.005 & 0.29 & 0 & 0.99 & 1.00 & 0.56 & 1.76 \\
\hline Remaining sources & -1.2 & 0.21 & 32.47 & $<0.0001$ & 0.30 & 0.20 & 0.46 \\
\hline Delay in management & 1.47 & 0.17 & 78.53 & $<0.0001$ & 4.33 & 3.13 & 5.99 \\
\hline Age & 0.04 & 0.004 & 103.58 & $<0.0001$ & 1.04 & 1.04 & 1.05 \\
\hline Immunosuppression & 1.24 & 0.17 & 55.79 & $<0.0001$ & 3.46 & 2.5 & 4.79 \\
\hline Constant & -7.52 & 0.41 & 342.24 & $<0.0001$ & 0.001 & & \\
\hline
\end{tabular}

\section{$O R$ odds ratio}

${ }^{\mathrm{a}}$ Compared with small bowel perforation 
Table 4 Direct logistic regression model showing the ability of WSES Sepsis Severity Score in predicting mortality of patients complicated intra-abdominal infection, global study of 132 centres, $(n=4553)$

\begin{tabular}{lrrrrrrr}
\hline Variable & B & S.E. & Wald & $P$ value & OR & \multicolumn{2}{c}{ OR 95 \% C.I. } \\
\cline { 6 - 9 } & & & & & & Lower & Upper \\
\hline WSESSCORE & 0.58 & 0.02 & 639.59 & $<0.0001$ & 1.784 & 1.706 & 1.866 \\
Constant & -5.79 & 0.19 & 958.74 & $<0.0001$ & .003 & & \\
\hline
\end{tabular}

OR odds ratio

predictors of mortality. Accordingly the ability of the score to predict mortality was tested by a direct logistic regression which is shown in Table 4. Again, this model using only the sepsis severity score was highly significant $(p<0.0001, \mathrm{R} 2=0.5)$. The odds of death increased by 0.78 by an increase on one score which is remarkable.

Figure 1 shows that WSES Sepsis Severity Score had a very good ability of distinguishing those who survived from those who died. The overall mortality rate was $9.2 \%$ (416/4533). This was $0.63 \%$ for those who had a score of $0-3,6.3 \%$ for those who had a score of $4-6$, and $41.7 \%$ for those who had a score of $\geq 7$. The receiver operating characteristic curve showed that the best cutoff point for predicting mortality was a Sepsis Severity Score. 5.5 was the best predictor of mortality having a sensitivity of $89.2 \%$, a specificity of $83.5 \%$ and a positive likelihood ratio of 5.4 (Fig. 2).

\section{Discussion}

Complicated intra-abdominal infections remain an important source of patient morbidity and may be frequently associated with poor clinical prognosis. Treatment of patients with cIAIs, has been usually described to achieve satisfactory results if adequate management is established [15]. However, results from published clinical trials may not be representative of the true morbidity and mortality rates of such severe infections. First of all, patients who have perforated appendicitis are usually over-represented in clinical trials. Furthermore patients with intraabdominal infection enrolled in clinical trials have often an increased likelihood of cure and survival. In fact the trial eligibility criteria usually restrict the inclusion of patients with co-morbid diseases that would increase the death rate of patients with intraabdominal infections [16]. In the WISS study we enrolled all the patients older than 18 years old with complicated intra-abdominal infections in the studyperiod and the overall mortality rate was $9.2 \%(416 /$ 4533). Stratification of the patient's risk is essential in order to optimize the treatment plan. Patients with intra-abdominal infections are generally classified into low risk and high risk. "High risk" is generally intended to describe patients with a high risk for treatment failure and mortality. In high risk patients the increased mortality associated with inappropriate management cannot be reversed by subsequent modifications. Therefore early prognostic

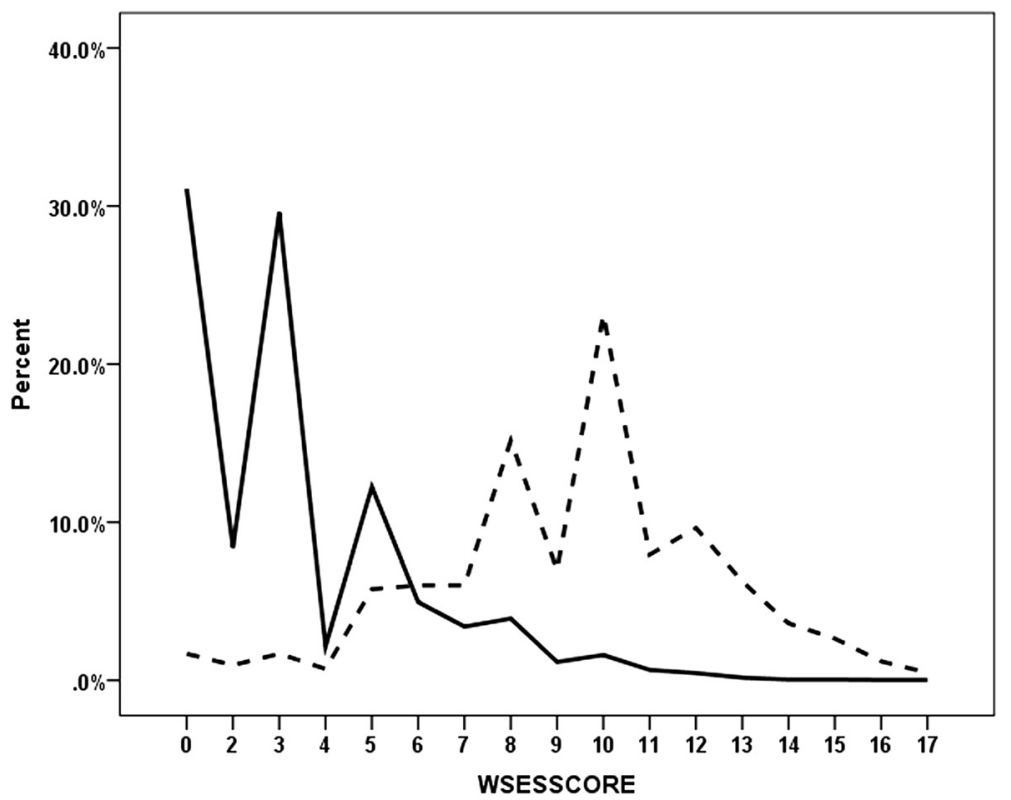

Fig. 1 Distribution of the percentile WSES Sepsis Severity Score of complicated intra-abdominal infection patients for those who survived (solid line) $(n=4117)$ and those who died (interrupted line) $(n=416)$ 


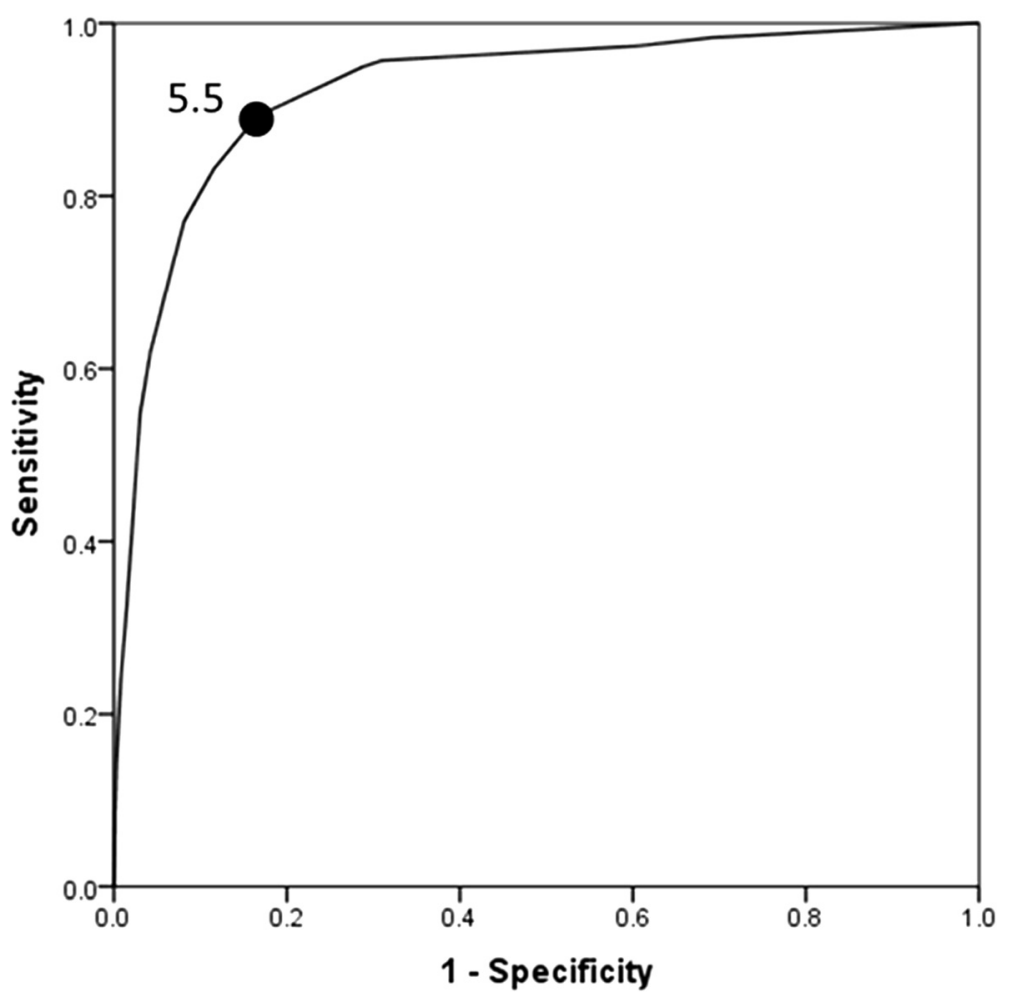

Fig. 2 Receiver operating characteristic curve for the best WSES Sepsis Severity Score that predicted mortality in patients having complicated intra-abdominal infection, global study of 132 centres, $(n=4553)$

evaluation of complicated intra-abdominal infections is important to assess the severity and decide the aggressiveness of treatment.

Scoring systems can be roughly divided into two groups: disease-independent scores for evaluation of serious patients requiring care in the intensive care unit (ICU) such as APACHE II and Simplified Acute Physiology Score (SAPS II) and peritonitis-specific scores such as Mannheim Peritonitis Index (MPI) [17].

Although considered a good marker, APACHE II value in peritonitis has been questioned because of the difficulty of the APACHE II to evaluate interventions despite the fact that interventions might significantly alter many of the physiological variables. Moreover it requires appropriate software to be calculated [18].

The MPI is specific for peritonitis and easy to calculate. MPI was designed by Wacha and Linder in 1983 [19]. It was based on a retrospective analysis of data from 1253 patients with peritonitis. Among 20 possible risk factors, only 8 proved to be of prognostic relevance and were entered into the Mannheim Peritonitis Index, classified according to their predictive power. After 30 years, identifying a new clinical score to assess the severity the cIAIS would be clinically relevant in order to modulate the aggressiveness of treatment according the type of infection and the clinical characteristics of the patients.
WSES Sepsis Severity Score is a new practical clinical severity score for patients with complicated intraabdominal infections. It is specific for cIAIs and easy to calculate, even during surgery. It may be relevant in order to modulate the aggressiveness of treatment particularly in higher risk patients.

The score is illustrated in Appendix. The statistical analysis shows that the sepsis severity score has a very good ability of distinguishing those who survived from those who died. The overall mortality was $0.63 \%$ for those who had a score of $0-3,6.3 \%$ for those who had a score of $4-6,41.7 \%$ for those who had a score of $\geq 7$. In patients who had a score of $\geq 9$ the mortality rate was $55.5 \%$, those who had a score of $\geq 11$ the mortality rate was $68.2 \%$ and those who had a score $\geq 13$ the mortality rate was $80.9 \%$

\section{Conclusions}

Given the sweeping geographical distribution of the participating medical centers, WSES Sepsis Severity Score for patients with complicated Intra-abdominal infections can be used on global level. It has shown high sensitivity, specificity, and likelihood ratio that may help us in making clinical decisions. 


\section{Appendix}

Table 5 WSES sepsis severity score for patients with complicated Intra-abdominal infections (Range: 0-18)

\begin{tabular}{|c|c|}
\hline \multicolumn{2}{|l|}{ Clinical condition at the admission } \\
\hline $\begin{array}{l}\text { - Severe sepsis (acute organ dysfunction) at the } \\
\text { admission }\end{array}$ & 3 score \\
\hline $\begin{array}{l}\text { - Septic shock (acute circulatory failure characterized } \\
\text { by persistent arterial hypotension. It always requires } \\
\text { vasopressor agents) at the admission }\end{array}$ & 5 score \\
\hline \multicolumn{2}{|l|}{ Setting of acquisition } \\
\hline - Healthcare associated infection & 2 score \\
\hline \multicolumn{2}{|l|}{ Origin of the IAls } \\
\hline - Colonic non-diverticular perforation peritonitis & 2 score \\
\hline - Small bowel perforation peritonitis & 3 score \\
\hline - Diverticular diffuse peritonitis & 2 score \\
\hline - Post-operative diffuse peritonitis & 2 score \\
\hline \multicolumn{2}{|l|}{ Delay in source control } \\
\hline $\begin{array}{l}\text { - Delayed initial intervention [Preoperative duration of } \\
\text { peritonitis (localized or diffuse) }>24 \text { h)] }\end{array}$ & 3 score \\
\hline \multicolumn{2}{|l|}{ Risk factors } \\
\hline - Age $>70$ & 2 score \\
\hline $\begin{array}{l}\text { - Immunosuppression (chronic glucocorticoids, } \\
\text { immunosuppresant agents, chemotherapy, lymphatic } \\
\text { diseases, virus) }\end{array}$ & 3 score \\
\hline
\end{tabular}

\section{Competing interests}

The authors declare that they have no competing interests.

\section{Authors' contributions}

MS designed the study and wrote the manuscript. FMA-Z performed statistical analysis. All authors participated in the study. All authors read and approved the final manuscript.

\section{Author details}

${ }^{1}$ Department of Surgery, Macerata Hospital, Macerata, Italy. ${ }^{2}$ Department of Surgery, College of Medicine and Health Sciences, UAE University, Al-Ain, United Arab Emirates. ${ }^{3}$ Department of Emergency Surgery, Maggiore Hospital, Parma, Italy. ${ }^{4}$ General and Upper Gl Surgery, Queen Elizabeth Hospital, Birmingham, UK. ${ }^{5}$ Department of Surgery, Maggiore Hospital, Bologna, Italy. ${ }^{6}$ Department of Surgery, UC San Diego Medical Center, San Diego, USA. ${ }^{7}$ Fundación Valle del Lili, Universidad del Valle, Cali, Colombia. ${ }^{8}$ Abdominal Center, University Hospital Meilahti, Helsinki, Finland. ${ }^{9}$ Division of Trauma Surgery, Hospital de Clinicas, School of Medical Sciences, University of Campinas, Campinas, Brazil. ${ }^{10}$ General and Emergency Surgery, Papa Giovanni XXIII Hospital, Bergamo, Italy. ${ }^{11}$ General Surgery, ULSS19 del Veneto, Adria, RO, Italy. ${ }^{12}$ Department of Surgery, Mansoura University Hospital, Mansoura, Egypt. ${ }^{13}$ Department of General Surgery, Al Ain Hospital, Al-Ain City, United Arab Emirates. ${ }^{14}$ Department of Surgery, Kwara State General Hospital, llorin, Nigeria. ${ }^{15}$ Department of Surgery, Ahmadu Bello University Teaching Hospital Zaria, Kaduna, Nigeria. ${ }^{16}$ Department of Surgery, LAUTECH Teaching Hospital, Osogbo, Nigeria. ${ }^{17}$ Department of General Surgery, Training and Research Hospital of Mustafa Kemal University, Hatay, Turkey.

${ }^{18}$ Depatment of Surgery, King Fahad Medical City, Riyadh, Saudi Arabia. ${ }^{19}$ Primary Health Care Centre of Kissamos, Chania, Greece. ${ }^{20}$ Department of Surgery, University Hospital Center, Zagreb, Croatia. ${ }^{21}$ Clinical and Experimental Surgery, Brescia Civil Hospital, Brescia, Italy. ${ }^{22}$ Trauma and Acute Care Surgery Unit, Hadassah Hebrew University Medical Center, Jerusalem, Israel. ${ }^{23}$ Department of Surgery, Bizerte Hospital, Bizerte, Tunisia.

${ }^{24}$ Department of General Surgery, Istanbul Training and Research Hospital,
Istanbul, Turkey. ${ }^{25}$ Surgical II Division, S. Chiara Hospital, Trento, Italy. ${ }^{26}$ Department of General Surgery, Hospital San Juan de Dios de La Serena, La Serena, Chile. ${ }^{27}$ Department of General and Emergency Surgery, SG Bosco Hospital, Turin, Italy. ${ }^{28}$ Emergency Surgery Department, 1st Municipal Hospital, Novosibirsk State Medical University, Novosibirsk, Russian Federation. ${ }^{29}$ Department of Surgery, University Hospital of Larissa, Larissa, Greece. ${ }^{30}$ Visceral Surgery, CHU, Angers, France. ${ }^{31}$ Chirurgia Generale, Ospedale di Città di Castello, Città di Castello, Italy. ${ }^{32}$ Department of Emergency Surgery, Umberto I Hospital, "La Sapienza" University, Rome, Italy. ${ }^{33}$ Department of Surgery, Kuala Krai Hospital, Kelantan, Malaysia. ${ }^{34}$ Department of Surgery, Regional Hospital, Limbe, Cameroon. ${ }^{35}$ General and Emergency Surgery, Policlinico Paolo Giaccone, Palermo, Italy. ${ }^{36}$ Department of General Surgery, Samsun Education and Research Hospital, Samsun, Turkey. ${ }^{37}$ Department of General and Digestive Tract Surgery, Alicante University General Hospital, Alicante, Spain. ${ }^{38}$ Department of Surgery, CHVNG/E, EPE, Vila Nova de Gaia, Portugal. ${ }^{39}$ Department of Surgery, Tianjin Nankai Hospital, Tianjin, China. ${ }^{40}$ Department of General Surgery, Emergency Municipal Hospital Pascani, Pascani, lasi, Romania. ${ }^{41}$ Department of Surgery, Numune Training and Research Hospital, Adana, Turkey. ${ }^{42}$ Department of Surgery, University Clinical Center, Tuzla, Bosnia and Herzegovina. ${ }^{43}$ Department General Surgery, Kipshidze Central University Hospital, Tbilisi, Georgia. ${ }^{44}$ Department of Surgery, Hamad General Hospital, Doha, Qatar. ${ }^{45}$ Department of Surgery, Riga East Clinical University Hospital, Riga, Latvia. ${ }^{46}$ Department of Surgery, Bahrain Defence Force Hospital, Manama, Bahrain. ${ }^{47}$ King Fahad Medical City, Riyadh, Saudi Arabia. ${ }^{48}$ Division of General and Emergency Surgery, Hospital Estadual Mario Covas, ABC School of Medicine, Santo André, Brazil. ${ }^{49}$ Department of Surgery 1, Vladimir City Clinical Hospital of Emergency Medicine, Vladimir, Russian Federation. ${ }^{50}$ Cirugía General y Digestiva, Hospital Universitario Miguel Servet, Zaragoza, Spain. ${ }^{51}$ 2nd Department of Surgery, Aretaieio University Hospital, Athens, Greece. ${ }^{52}$ Department of Surgery, Hospital Universitário Terezinha de Jesus, Faculdade de Ciências Médicas e da Saúde de Juiz de Fora, Juiz de Fora, Brazil. ${ }^{53}$ Department of Surgery, Hospital Escola Padre Albino, Catanduva, Brazil. ${ }^{54}$ Department of Surgery, Ascoli Piceno Hospital, Ascoli Piceno, Italy. ${ }^{55}$ Department of General Surgery, Trabzon Kanuni Training and Research Hospital, Trabzon, Turkey. ${ }^{56}$ Department of Surgery, Government Medical College and Hospital, Chandigarh, India. ${ }^{57}$ Hospital Universitario del Valle, Universidad del Valle, Cali, Colombia. ${ }^{58}$ 2nd Surgical Department of Medical Faculty Comenius University, University Hospital Bratislava, Bratislava, Slovakia. ${ }^{59}$ 2nd Surgical Department, General Hospital of Kavala, Kavala, Greece. ${ }^{60}$ Department of Surgery, Mengucek Gazi Training Research Hospital, Erzincan, Turkey. ${ }^{61}$ Department of Emergency and Critical Care Medicine, Jichi Medical University, Shimotsuke, Japan. ${ }^{2}$ Department of Surgery, S M S Hospital, Jaipur, India. ${ }^{63}$ Department of Surgery, Hospital of Lithuanian University of Health Sciences, Kaunas, Lithuania. ${ }^{64} \mathrm{Clinic}$ for Emergency Surgery, Medical Faculty University of Belgrade, Belgrade, Serbia. ${ }^{65}$ Division Digestive Surgery and Urology, Turku University Hospital, Turku, Finland. ${ }^{66} 3$ rd Department of General Surgery, Jagiellonian Univeristy Collegium Medium, Kraków, Poland. ${ }^{67}$ Department of Emergency Surgery, City Hospital, Mozyr, Belarus. ${ }^{68}$ Department of Surgery, Ilsan Paik Hospital, Inje University College of Medicine, Goyang, Republic of Korea. ${ }^{69}$ Department of Surgery, Edendale Hospital, Pietermaritzburg, South Africa. ${ }^{70}$ Department of Surgery, University Clinical Center of Kosovo, Pristina, Kosovo. ${ }^{71}$ Department of General Surgery, Krishna Hospital, Karad, India. ${ }^{72}$ Department of General Surgery, Republican Vilnius University Hospital, Vilnius, Lithuania. ${ }^{73}$ Department of Surgery, York Teaching Hospital NHS Foundation Trust, York, UK. ${ }^{74}$ General Surgery/Coloretal Unit, Braga Hospital, Life and Health Sciences Research Institute (ICVS), School of Health Sciences, University of Minho, Braga, Portugal. ${ }^{75}$ Department of Surgery, Yonsei University College of Medicine, Seoul, South Korea. ${ }^{76}$ Department of Surgery, Hospital La Paz, Madrid, Spain. ${ }^{77}$ Cirugía de Urgencias, Hospital Universitario Donostia, Donostia, Spain. ${ }^{78}$ Department of Surgery, Faculty of Medicine Siriraj Hospital, Mahidol University, Bangkok, Thailand. ${ }^{79}$ Department of Surgery, Insular University Hospital of Gran Canaria, Las Palmas, Spain. ${ }^{80} 1$ st Department of Surgery, Kavala General Hospital, Kavala, Greece. ${ }^{81}$ Department of General Surgery, Mysore Medical College and Research Institute, Government Medical College Hospital Mysore, Mysore, India. ${ }^{82}$ 2nd Department of Surgery, Jagiellonian University Medical College, Krakow, Poland. ${ }^{83}$ First Department of Surgery, Tzaneio Hospital, Piraeus, Greece. ${ }^{84}$ Department of General Surgery and Surgical Oncology, Le Scotte Hospital, Siena, Italy. ${ }^{85}$ Department of Surgery, University Hospital, Valencia, Spain. ${ }^{86}$ Department of Surgery, 
Post-Graduate Institute of Medical Sciences, Rohtak, India. ${ }^{87}$ Department of Surgery, Radiology, University Hospital of the West Indies, Kingston, Jamaica. ${ }^{88}$ General Surgery Department, Centro Hospitalar de São João, Porto, Portugal. ${ }^{89}$ Second Surgical Clinic, Emergency Hospital of Craiova, Craiova, Romania. ${ }^{90}$ 3rd Department of Surgery, Haepa University Hospital, Thessaloniki, Greece. ${ }^{91}$ Department of Surgery, CH Armentieres, Arras, France. ${ }^{92}$ Surgery Department, University Hospital Hassan II, Fez, Morocco. ${ }^{93}$ Department of Surgery, Onandjokwe Hospital, Ondangwa, Namibia. ${ }^{94}$ Department of Surgery, Emergency Hospital of Bucharest, Bucharest, Romania. ${ }^{95}$ Department of General Surgery, Lewisham \& Greenwich NHS Trust, London, UK. ${ }^{96}$ Department of Surgery, University of Ilorin Teaching Hospital, Ilorin, Nigeria. ${ }^{97}$ Department of Surgery, King Abdalla University Hospital, Irbid, Jordan. ${ }^{98}$ Department of Surgery, Elazig Training and Research Hospital, Elazig, Turkey. ${ }^{99}$ Department of Laparoscopic and Metabolic Surgery, Sunrise Hospital, Kochi, India. ${ }^{100}$ Department of Surgery, Sant'Antonio Abate Hospital, Gallarate, Italy. ${ }^{101}$ Division of Emergency and Trauma Surgery, Ribeirão Preto Medical School, Ribeirão Preto, Brazil. ${ }^{102}$ Surgery 1 Unit, Centro Hospitalar Tondela Viseu, Viseu, Portugal. ${ }^{103}$ Department of Surgery, UMC Ljubljana, Ljubljana, Slovenia. ${ }^{104}$ Department of Surgery, Bharati Medical College and Hospital, Sangli, India. ${ }^{105}$ Department of Surgery, Insubria University Hospital, Varese, Italy. ${ }^{106}$ Abdominal and General Surgery Department, General Hospital Jesenice, Jesenice, Slovenia.

${ }^{107}$ Department of Surgery, Hospital de Alta Especialidad de Veracruz, Veracruz, Mexico. ${ }^{108}$ General Surgery Department, Medical University, University Hospital St George, Plovdiv, Bulgaria. ${ }^{109}$ Department of Surgery, Fundación Jimenez Díaz, Madrid, Spain. ${ }^{110}$ Department of Surgery, Good Hope Hospital, Heart of England NHS Foundation Trust, Birmingham, UK. ${ }^{111}$ Department of General Surgery, Tan Tock Seng Hospital, Novena, Singapore. ${ }^{112}$ Departement of Surgery, Fatabenefratelli Isola Tiberina Hspital, Rome, Italy. ${ }^{113}$ Department of Surgery, Hospital and Comprehensive Cancer Centre Novy Jicin, Novy Jicin, Czech Republic. ${ }^{114}$ Department of General Surgery, Clinical Hospital at Chelyabinsk Station of OJSC "Russian Railroads", Chelyabinsk, Russian Federation. ${ }^{115}$ 3th Department of Surgery, laso General Hospital, Athens, Greece. ${ }^{116}$ Department of Surgery, North Estonia Medical Center, Tallin, Estonia. ${ }^{117}$ Department of Surgery, Baskent University Ankara Hospital, Ankara, Turkey. ${ }^{118}$ General Surgery Service, Trauma University Hospital, Tirana, Albania. ${ }^{119} 1$ st Department of Surgery - Department of Abdominal, Thoracic Surgery and Traumatology, General University Hospital, Prague, Czech Republic. ${ }^{120}$ Department of General Surgery, Sakarya Teaching and Research Hospital, Sakarya, Turkey. ${ }^{121}$ Department of Renal and Pancreas Transplantation, Manchester Royal Infirmary, Manchester, UK. ${ }^{122}$ Department of Surgery, Red Cross Hospital, Beverwijk, Netherlands. ${ }^{123}$ Emergency Surgery, Arcispedale S.Anna Azienda Ospedaliero-Universitaria di Ferrara, Ferrara, Italy. ${ }^{124}$ Department of Surgery, Medical School University Pecs, Pecs, Hungary. ${ }^{125}$ Department of Surgery, Montichiari Hospital, Ospedali Civili Brescia, Brescia, Italy. ${ }^{126} 1$ st Surgical Clinic, St. Spiridon Hospital, lasi, Romania. ${ }^{127}$ Department of Surgery, Rajendra Institute of Medical Sciences, Ranchi, India. ${ }^{128}$ Department of Surgery, Kocaeli University Training and Research Hospital, Kocaeli, Turkey. ${ }^{129}$ Trauma and Emergency Surgery Department, Chang Gung Memorial Hospital, Taoyuan City, Taiwan. ${ }^{130}$ Department of Surgery, MOSC Medical College Kolenchery, Cochin, India. ${ }^{131}$ General and Digestive Surgery Department, Teaching Hospital Yalgado Ouedraogo, Ouagadougou, Burkina Faso.

\section{Received: 17 November 2015 Accepted: 10 December 2015}

Published online: 16 December 2015

\section{References}

1. Sartelli M, Viale P, Catena F, Ansaloni L, Moore E, Malangoni M, et al. 2013 WSES guidelines for management of intra-abdominal infections. World J Emerg Surg. 2013;8(1):3.

2. Mulari K, Leppäniemi A. Severe secondary peritonitis following gastrointestinal tract perforation. Scand J Surg. 2004;93(3):204-8.

3. Horiuchi A, Watanabe Y, Doi T, Sato K, Yukumi S, Yoshida M, et al. Evaluation of prognostic factors and scoring system in colonic perforation. World J Gastroenterol. 2007;13(23):3228-31.

4. Evans HL, Raymond DP, Pelletier SJ, Crabtree TD, Pruett TL, Sawyer KG. Tertiary peritonitis (recurrent diffuse or localized disease) is not an independent predictor of mortality in surgical patients with intra-abdominal infection. Surg Infect. 2001;2:255-65.
5. McLauchlan GJ, Anderson ID, Grant IS, Fearon KCH. Outcome of patients with abdominal sepsis treated in an intensive care unit. Br J Surg. 1995;82:524-9.

6. Koperna T, Schulz F. Prognosis and treatment of peritonitis: Do we need new scoring systems? Arch Surg. 1996;131:180-6.

7. Pacelli F, Doglietto GB, Alfieri S, Piccioni E, Sgadari A, Gui D, et al. Prognosis in intra-abdominal infections. Multivariate analysis on 604 patients. Arch Surg. 1996;131:641-5.

8. Ohmann C, Yang Q, Hau T, Wacha H, the Peritonitis Study Group of the Surgical Infection Society Europe. Prognostic modelling in peritonitis. Eur J Surg. 1997;163:53-60.

9. Montravers P, Gauzit R, Muller C, Marmuse JP, Fichelle A, Desmonts JM. Emergence of antibiotic-resistant bacteria in cases of peritonitis after intraabdominal surgery affects the efficacy of empirical antimicrobial therapy. Clin Infect Dis. 1996;23:486-94.

10. Prabhu $V$, Shivani A. An overview of history, pathogenesis and treatment of perforated peptic ulcer disease with evaluation of prognostic scoring in adults. Ann Med Health Sci Res. 2014;4(1):22-9.

11. Sartelli M, Catena F, Ansaloni L, Leppaniemi A, Taviloglu K, van Goor H, et al. Complicated intra-abdominal infections in Europe: a comprehensive review of the CIAO study. World J Emerg Surg. 2012;7(1):36.

12. Sartelli M, Catena F, Ansaloni L, Coccolini F, Corbella D, Moore EE, et al. Complicated intra-abdominal infections worldwide: the definitive data of the CIAOW Study. World J Emerg Surg. 2014;9:37.

13. Sartelli M. A focus on intra-abdominal infections. World J Emerg Surg. 2010:5:9.

14. Dellinger RP, Levy MM, Rhodes A, Annane D, Gerlach H, Opal SM, et al. Surviving Sepsis Campaign Guidelines Committee including The Pediatric Subgroup. Surviving Sepsis Campaign: international guidelines for management of severe sepsis and septic shock, 2012. Intensive Care Med. 2013;39(2):165-228.

15. Mazuski JE, Solomkin JS. Intra-abdominal infections. Surg Clin North Am. 2009;89(2):421-37.

16. Merlino Jl, Malangoni MA, Smith CM, Lange RL. Prospective randomized trials affect the outcomes of intraabdominal infection. Ann Surg. 2001;233(6):859-66.

17. Komatsu S, Shimomatsuya T, Nakajima M, Amaya H, Kobuchi T, Shiraishi S, et al. Prognostic factors and scoring system for survival in colonic perforation. Hepatogastroenterology. 2005;52:761-64.

18. Koperna T, Semmler D, Marian F. Risk stratification in emergency surgical patients: is the APACHE II score a reliable marker of physiological impairment? Arch Surg. 2001;136(1):55-9.

19. Wacha H, Linder MM, Feldman U, Wesch G, Gundlach E, Steifensand RA. Mannheim peritonitis index - prediction of risk of death from peritonitis: construction of a statistical and validation of an empirically based index. Theor Surg. 1987;1:169-77.

\section{Submit your next manuscript to BioMed Central and we will help you at every step:}

- We accept pre-submission inquiries

- Our selector tool helps you to find the most relevant journal

- We provide round the clock customer support

- Convenient online submission

- Thorough peer review

- Inclusion in PubMed and all major indexing services

- Maximum visibility for your research

Submit your manuscript at www.biomedcentral.com/submit
Biomed Central 